\title{
A NOTE ON POINTWISE NONWANDERING TRANSFORMATIONS
}

\author{
W. H. GOTTSCHALK
}

Let $X$ be a $T_{1}$-space and let $f$ be a continuous transformation of $X$ into $X$ In the terminology of G. D. Birkhoff $[1$, p. 191 $],{ }^{1}$ a point $x$ of $X$ is said to be nonwandering under $f$ provided that to each neighborhood $U$ of $x$ there correspond infinitely many positive integers $n$ for which $U \cap f^{n}(U) \neq \varnothing$; also, the transformation $f$ is said to be pointwise nonwandering provided that each point of $X$ is nonwandering under $f$.

THEOREM 1. If $f$ is pointwise nonwandering, then so also is $f^{\text {h }}$ for every positive integer $k$.

Proof. (We make use of a technique employed by Erdös and Stone [2, pp. 126-127].) Suppose $k$ is a positive integer, $x_{0} \in X$, and $U_{0}$ is a neighborhood (= open neighborhood) of $x_{0}$. Let $n_{1}$ be a positive integer for which $U_{0} \cap f^{n_{1}}\left(U_{0}\right) \neq \varnothing$. Choose $x_{1} \in U_{0}$ so that $f^{n_{1}}\left(x_{1}\right) \in U_{0}$ and a neighborhood $U_{1}$ of $x_{1}$ so that $U_{1} \subset U_{0}$ and $f^{n_{1}}\left(U_{1}\right) \subset U_{0}$. Let $n_{2}$ be a positive integer for which $U_{1} \cap f^{n_{2}}\left(U_{1}\right) \neq \varnothing$. Choose $x_{2} \in U_{1}$ so that $f^{n_{2}}\left(x_{2}\right) \in U_{1}$ and a neighborhood $U_{2}$ of $x_{2}$ so that $U_{2} \subset U_{1}$ and $f^{n_{2}}\left(U_{2}\right) \subset U_{1}$. Continuing this process, we obtain a sequence $\left\{n_{i}\right\}$ of positive integers and a sequence $\left\{U_{i}\right\}$ of neighborhoods so that $U_{i} \subset U_{i-1}$ and $f^{n_{i}}\left(U_{i}\right) \subset U_{i-1}(i=1,2, \cdots)$. Let $r_{i}$ denote the integer for which $1 \leqq r_{i} \leqq k$ and $n_{i} \equiv r_{i} \bmod k$. Infinitely many of the $r_{i}$ are equal to some integer, say $r$. We may suppose $r_{i}=r, U_{i} \subset U_{i-1}$ and ${ }^{n_{i}}\left(U_{i}\right) \subset U_{i-1}(i=1,2, \cdots)$. Choose an arbitrary positive integer $p$. Define $n=\sum_{i=1}^{p k} n_{i}$. Now $n \equiv 0 \bmod k$. Choose $x \in U_{p k}$. Clearly, $x \in U_{0}$ and $f^{n}(x) \in U_{0}$. Hence, $U_{0} \cap f^{n}\left(U_{0}\right) \neq \varnothing$. Since $n \geqq p$, the proof is completed.

Lemma 1. If $f(X)=X$ is a homeomorphism, if $A$ and $B$ are closed connected sets for which $A \cup B=X, A \cap B=x \in X$ and $A \cap f(A) \neq \varnothing$ $\neq B \cap f(B)$, and if $x$ is nonwandering, then $x$ is fixed.

Proof. Assume $x$ is not fixed. We may suppose that $f(x) \in B$. Now $x \notin f^{-1}(A)$ for in the contrary case $f(x) \in A \cap B=x$. The set $f(A)$ is connected and intersects both $A$ and $B$. Hence, $x \in f(A)$. There exists a neighborhood $U$ of $x$ such that $U \cap f^{-1}(A)=\varnothing$ and such that 1946

Presented to the Society, August 14, 1944; received by the editors February 1,

${ }^{1}$ Numbers in brackets refer to the bibliography at the end of the paper. 
$U \subset f(A)$ for if the latter condition could not be satisfied, $x \in f(B)$ and $f(x)=f(A \cap B)=f(A) \cap f(B) \ni x$. Now $A \subset f(A)$ since otherwise $A \cap f(B) \neq \varnothing \neq B \cap f(B)$ whence $x \in f(B)$ and as before $f(x)=x$. Thus $A \subset f^{n}(A)$ for each integer $n \geqq 1$. We conclude that for each integer $n \geqq 1, f^{n+1}(U) \cap f^{n}(A)=\varnothing$ and, since also $U \subset f(A) \subset f^{n}(A), U \cap f^{n+1}(U)$ $=\varnothing$. This contradicts the hypothesis that $x$ is nonwandering.

Theorem 2. If $X$ is connected and if $f(X)=X$ is a pointwise nonwandering homeomorphism, then each cut point of $X$ is periodic.

Proof. Let $x$ be a cut point of $X$. There exist closed connected sets $A$ and $B$ such that $A \cup B=X, A \cap B=x$ and $A \neq x \neq B$. The proof is split into two exhaustive cases. Case I: Either $A \cap f^{i}(A) \neq \varnothing(i=1$, $2, \cdots)$, or $B \cap f^{i}(B) \neq \varnothing(i=1,2, \cdots)$. Case II: There exist positive integers $m$ and $n$ such that $A \cap f^{m}(A)=\varnothing$ and $B \cap f^{n}(B)=\varnothing$.

Suppose Case I occurs. We may assume that $A \cap f^{i}(A) \neq \varnothing(i=1$, $2, \cdots)$. Since $B-x$ is open, there exists a positive integer $k$ such that $B \cap f^{k}(B) \neq \varnothing$. By Theorem $1, x$ is nonwandering under $f^{k}$ and by Lemma $1, x$ is therefore fixed under $f^{k}$.

Suppose Case II occurs. Now $B \supset f^{m}(A)$ and $A \supset f^{n}(B)$. Hence $f^{n}(B) \supset f^{m+n}(A)$ and $f^{m}(A) \supset f^{m+n}(B)$. It follows that $A \supset f^{m+n}(A)$ and $B \supset f^{m+n}(B)$. Thus, $f^{m+n}(x)=f^{m+n}(A \cap B)=f^{m+n}(A) \cap f^{m+n}(B) \subset A \cap B$ $=x$. (Actually Case II can never occur.)

Corollary 1. If $X$ is a compact connected semi-locally connected metric space and if $f(X)=X$ is a pointwise nonwandering homeomorphism, then every cyclic element of $X$ which is not an end point is periodic.

A theorem [3, p. 242] of Schweigert's shows that Corollary 1 permits a weakening of hypothesis from pointwise recurrence (= "pointwise almost periodicity" in the sense of Ayres) to pointwise nonwandering in certain theorems of Ayres and Whyburn on the behavior of cyclic elements under a homeomorphism. The reader is referred to Schweigert's paper [3] for complete references to the work of Ayres and Whyburn.

\section{BIBLIOGRAPHY}

1. G. D. Birkhoff, Dynamical systems, Amer. Math. Soc. Colloquium Publications, vol. 9, New York, 1927.

2. P. Erdös and A. H. Stone, Some remarks on almost periodic transformations, Bull. Amer. Math. Soc. vol. 51 (1945) pp. 126-130.

3. G. E. Schweigert, Fixed elements and periodic types for homeomorphisms on s. l. c. continua, Amer. J. Math. vol.66 (1944) pp. 229-244.

University of Pennsylvania 